Article

\title{
Interference-Aware Opportunistic Dynamic Energy Saving Mechanism for Wi-Fi Enabled IoTs
}

\author{
Il-Gu Lee ${ }^{\dagger}$ \\ Department of Convergence Security Engineering, Sungshin University, Seoul 02844, Korea; \\ iglee@sungshin.ac.kr or iglee19@gmail.com; Tel.: +82-292-071-45 \\ + Current address: 2, Bomun-ro 34da-gil, Seongbuk-gu, Seoul 02844, Korea.
}

Academic Editors: Sabah Mohammed and Chris Speed

Received: 7 June 2017; Accepted: 12 July 2017; Published: 18 July 2017

\begin{abstract}
The wireless local area network (WLAN) is one of the most popular wireless technologies offering connectivity today, and one of the candidates for the internet of things (IoTs). However, WLAN's inefficiency in terms of complexity and relatively large power consumption compared with other wireless standards has been reported as a major barrier for IoTs applications. This paper proposes an interference-aware opportunistic dynamic energy saving mechanism to improve energy efficiency for Wi-Fi enabled IoTs. The proposed scheme optimizes operating clock frequencies adaptively for signal processing when the mobile station transmits packets in partial sub-channels. Evaluation results demonstrate that the proposed scheme improves energy efficiency by approximately $34 \%$.
\end{abstract}

Keywords: dynamic energy saving; multi-channel; power consumption; WiFi; IoTs

\section{Introduction}

As demand for high data rate and low power mobile applications has increased, many efforts have been made to increase the data rate and energy efficiency in wireless communication systems. In order to obtain a high data rate and high reliability, wireless local area network (WLAN) chipsets got more complex and supported a wider channel bandwidth [1-3]. Since data is transmitted using a wider bandwidth to support high throughput, the required operating clocks of the circuits have had to be increased, which causes an increase in power consumption. In addition, the required energy efficiency levels of RF, analog and digital circuitry design are more constrained. However, battery technology has not kept up with these demands. Therefore, the energy budget of a wireless communication interface with a limited battery capacity is extremely limited because wireless communication devices are rapidly developing to accept additional elements and technologies to add new functions and increase the performance [4]. Accordingly, there is a desire for a method for using the limited battery capacity longer and more efficiently [5].

Since the battery capacity is limited in the mobile terminal or the sensor, a technology for minimizing energy consumption is necessary [6,7]. A wireless interface may reduce power consumption by two methods. First, the wireless interface is awake only when there is data to be exchanged with another wireless device [8]. Otherwise, a power save mode is maintained to reduce energy consumption. Second, when a received packet does not correspond to an identifier (ID) of the wireless interface, data processing is not performed to reduce energy consumption [1]. According to these conventional technologies, the energy efficiency may deteriorate when the mobile station is actively processing in the maximum operating clock mode of the circuits to accept the widest channel bandwidth signal. In particular, since the WLAN is required to perform high speed digital processing to support a high performance service, a high frequency clock is used and a large hardware size is required. In addition, since a relatively high voltage scale is used, power consumption 
is extremely high. However, this is not always true because mobile station adapts transmission bandwidth using dynamic bandwidth operation. In a typical WLAN chipset, it has analog and digital filters for 20/40/80 MHz signal bandwidths for IEEE 802.11ac, and 1/2/4/8 MHz signal bandwidths for IEEE 802.11ah [1,3]. Nyquist sampling theorem states that the minimum sampling-rate is two times to the signal bandwidth [9]. Therefore, the dynamic bandwidth operation is proposed to save power if the device needs to process a narrower signal bandwidth than the maximum operatable signal bandwidth.

WLANs have been a good solution for the internet of things (IoTs) because there are various IoTs applications which have different requirements, such as throughput, service coverage, and power consumption [8]. However, in IoTs networks, the dynamic channel access and heterogeneous network conditions lead to partial sub-channel operation, which is not the widest operatable channel bandwidth, so it is possible to optimize signal processing in the transceivers in order to improve its energy efficiency. For example, a typical WLAN supports $80 \mathrm{MHz}$ channel bandwidth operation, and it can support $20 \mathrm{MHz}, 40 \mathrm{MHz}$, or $80 \mathrm{MHz}$ bandwidth operation. Here, $20 \mathrm{MHz}$ and $40 \mathrm{MHz}$ channels are the sub-channels for $80 \mathrm{MHz}$ channel bandwidth operation. This paper proposes a dynamic power saving mechanism to improve energy efficiency for Wi-Fi enabled IoTs utilizing multiple sub-channels. The remainder of this paper is organized as follows. In Section 2, related works are described. In Section 3, a dynamic bandwidth operation is introduced and a dynamic power saving mechanism is proposed in Section 4. Section 5 presents the evaluation results, and finally Section 6 draws conclusions.

\section{Related Work}

For improving energy efficiency, unscheduled automatic power save delivery (U-APSD) was defined in the IEEE standard 802.11e [8], and it is also known as Wi-Fi Multi-Media (WMM) power save. While the stations (STAs) using legacy PSM need to wait for the beacon frame to initiate a data download, WMM-PS STAs can initiate the download at any time, thus allowing more frequent data transmission for applications that require them. Further, power save multi-poll (PSMP) is a feature of 802.11n that extends the APSD mechanism by allowing the STAs to operate on a group schedule rather than individually. In 802.11n, spatial multiplexing power save (SMPS) was defined to reduce idle listening power using request to send (RTS) and clear to send (CTS) to enable multiple antennas [8]. The legacy PSM is mandatorily supported in all WLAN devices because IEEE 802.11 must be backward compatible with legacy devices and access categories while WMM-PS, PSMP, and SMPS are optionally operated to improve power saving efficiency for specific uses, such as multiple-input multiple-output (MIMO), group schedule, and timing-sensitive applications. In the existing 802.11 WLAN standards, 802.11 legacy power saving mode (PSM) is operated by traffic existence only without considering network conditions, and so it incurs significant energy efficiency loss in a dynamic channel accessed network.

There have been several studies on the improvement of energy efficiency in the idle listening period. In [10], the authors present an adaptive clock rate during the idle listening period; this method reduces energy consumption by employing dummy bits or preambles. Combined with a carrier sensing indicator, the gated clock technique can be used for power saving to improve the dynamic energy efficiency at the receiver [11]. These techniques are very effective methods for reducing power consumption in the idle listening time. However, they cannot be used for data processing operation. The authors of [12] presented a performance characterization of 802.11ac in a testbed, and they showed that wider bandwidth improves throughput performance at the cost of higher power consumption.

\section{Dynamic Bandwidth Operation}

Therefore, heterogeneous standard terminals may be present in the same channels in adjacent locations or in adjacent channels. In such a condition, it is difficult to provide terminals that support a wide bandwidth with a high speed service due to interference from the same or adjacent channels. 
To solve the limit described above, a dynamic bandwidth allocation mechanism as shown in Figure 1 was introduced in the IEEE 802.11ac standard [1]. In an environment including a total of four sub-channels, channel bonding may be performed with respect to only two sub-channels meeting the condition, instead of all the four sub-channels, to transmit data. That is, when a sub-channel that is to be bonded for data transmission includes another signal, data transmission may be performed using the remaining channels and excluding the sub-channel.

Figure 2 illustrates the procedures for dynamic bandwidth allocation. The transmitter may transmit the RTS to the receiver through bonded multiple communication sub-channels. The receiver may receive the RTS transmitted from the transmitter. Then, the receiver may select at least one communication sub-channel from the multiple communication sub-channels that received the RTS. After receiving the RTS frames, the receiver may transmit the CTS to the transmitter as a response to the RTS with respect to the selected communication sub-channels. It should be noted that the sub-channel is selected by the receiver. The transmitter may receive the CTS transmitted from the receiver. Then, the transmitter may transmit a data packet to the transmitter through the selected communication sub-channels. It may use all or a subset of the communication channels that successfully received the CTS. The receiver may receive the data packet from the transmitter.

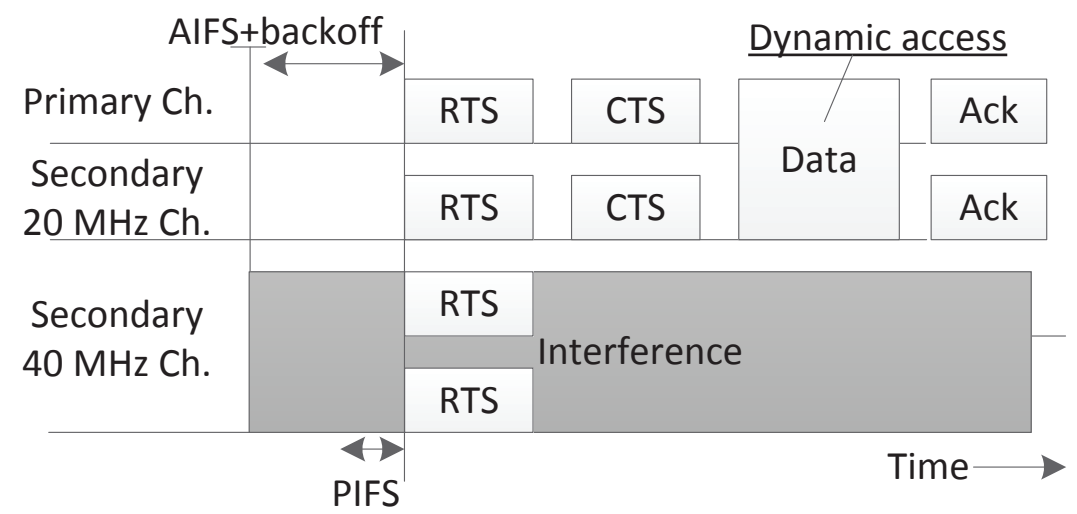

Figure 1. Dynamic bandwidth allocation mechanism; CTS: clear to send; RTS: request to send; Ack: acknowledgment; PIFS: point coordination function.

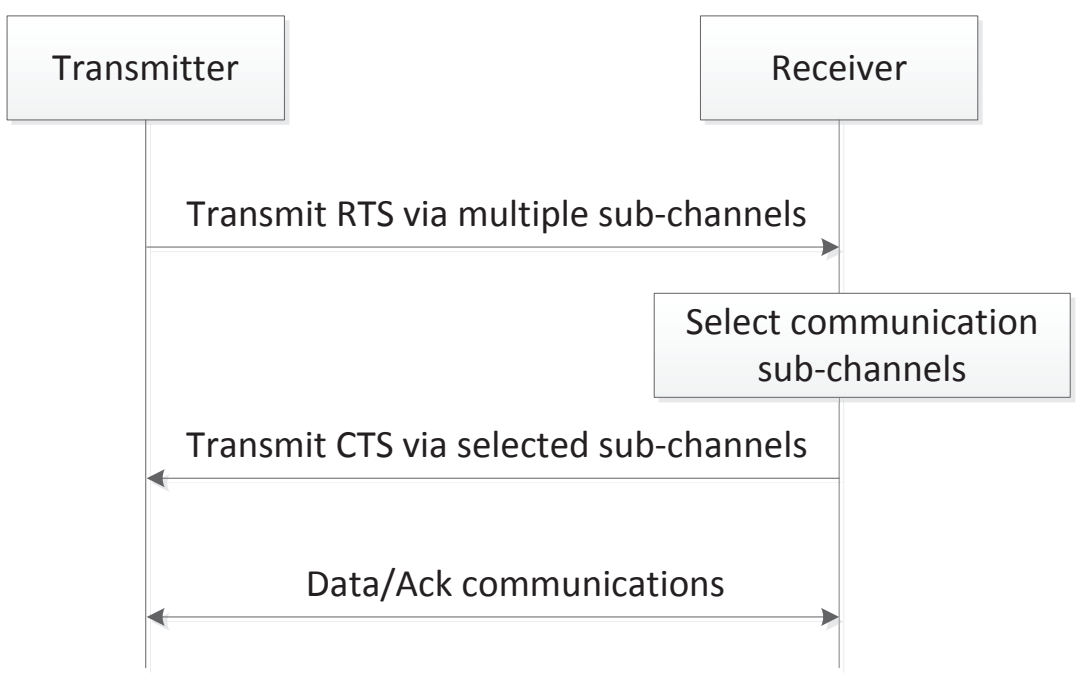

Figure 2. Flowchart for dynamic bandwidth allocation. 


\section{Proposed Dynamic Energy Saving Mechanism}

A wireless communication system consumes more power with a larger bandwidth because a larger bandwidth requires a higher processing speed, that is, the operation frequency, of a wireless communication circuit. Therefore, in this paper, we propose dynamic power saving mechanism to determine the communication sub-channels and operating clock frequencies to be performed based on the clear channel assessment (CCA) of each of the selected communication sub-channels. Figure 3 is a flowchart illustrating the proposed dynamic energy saving operation. The proposed method uses RTS/CTS frames for dynamic bandwidth operation between a transmitter and a receiver. At least one communication sub-channel is selected from the multiple communication sub-channels that have received the RTS, based on a CCA of set of communication channels. When all channels are affected by the interference signal and therefore the transmission PHY data rate is low, the absence of a good quality channel for high PHY data rate is noted through the CTS frame and the power save mode begins. As a result, the receiver selects the sub-channels and feedbacks the decision to transmitter, then the transmitter bonds the sub-channels that have received the CTS from the receiver. Here, the data packet is not transmitted on a communication channel that has not received the CTS among set of communication channels.

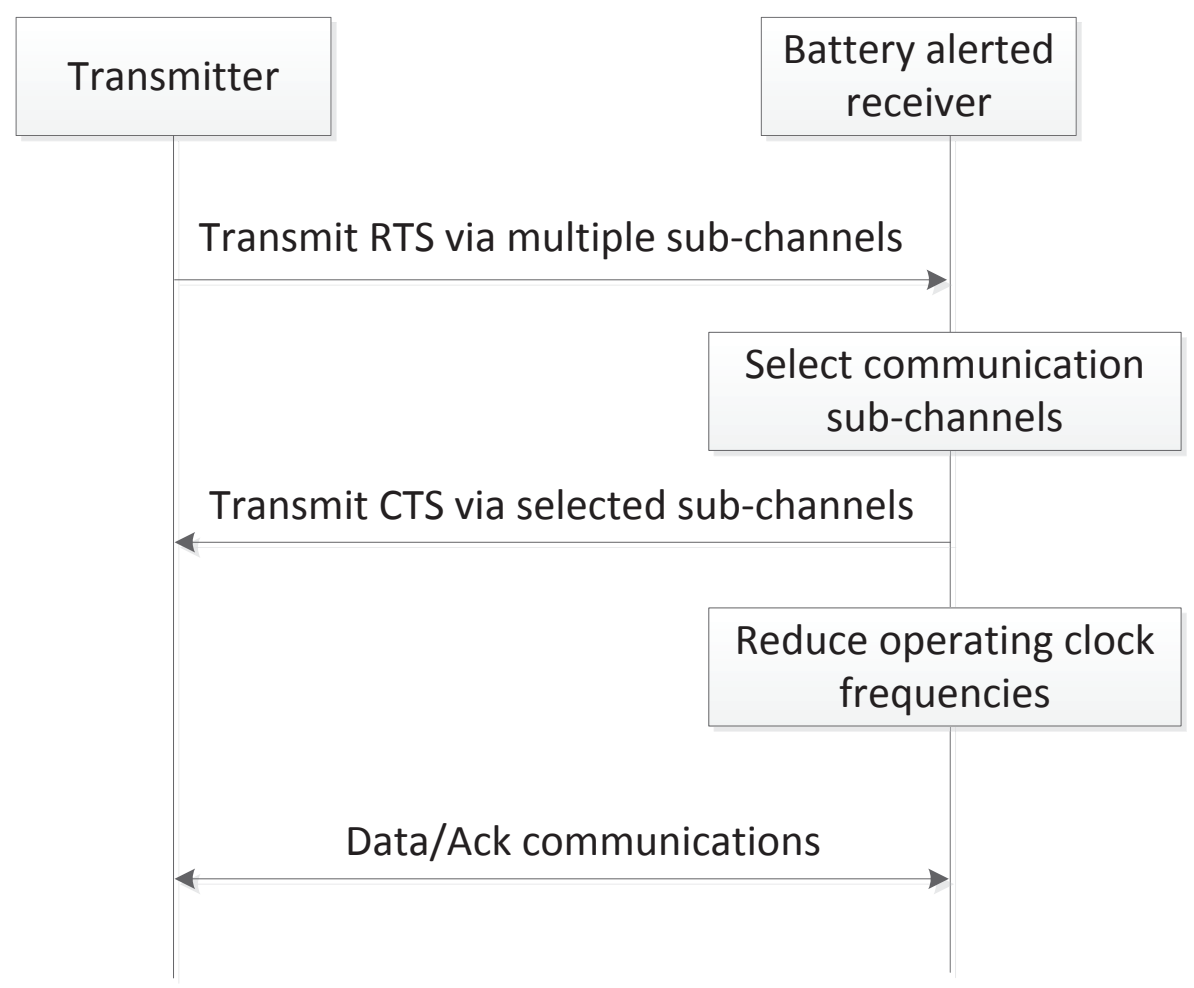

Figure 3. Flowchart for dynamic energy saving operation.

Figure 4 illustrates a multi-channel low power communication method according to the proposed energy saving operation. The left side of Figure 4 shows an example of the proposed energy saving mechanism that aggregates four bands and transmits RTS to four bands, but performs communication with only two bands. The receiver requests the energy saving operation through the dynamic bandwidth operation information field of the CTS. Thus, by transmitting the CTS, the receiver notes the transmitter reduction in channels and operating clock frequencies in order to save power. In the right side of Figure 4, there is a request to use a channel of a smaller width for transmission of a data packet during transmission of the CTS. During exchange of the RTS and CTS, the CCAs are measured. The power saving efficiency can be increased by using the RTS and the CTS in consideration of a 
battery capacity and the channel state of the wireless communication network. The main idea of the proposed dynamic energy saving mechanism is to improve the energy efficiency by reducing the operating clock frequency of ADC and digital front-end circuits including digital filters if the wireless system determines to use narrower channel bandwidth. Core digital modem maintains the operating clock frequency because clock rate converter transforms the clock rates between the digital front-end circuits and core digital modem. Thus, the transmission time is the same regardless the operating clock rate of ADC and digital front-end circuits. As the ADC and digital front-end circuits are major energy consumers in WLANs, the adaptive operating clock scheme is an effective way to save power.

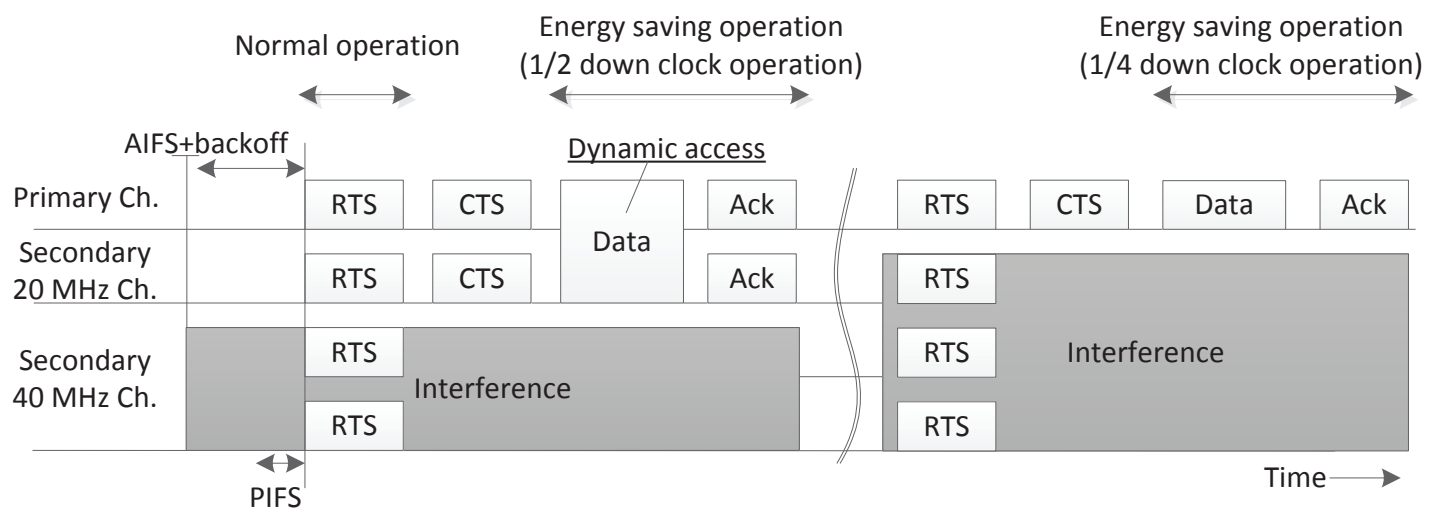

Figure 4. Dynamic energy saving operation; AIFS: arbitration interframe space.

\section{Performance Evaluation}

In dense networks, the energy consumption is an important criterion for designing and evaluating portable devices due to its impact on battery life. For evaluating the energy consumption of a wireless device, a typical energy consumption metric reflects the energy consumption measured in units of energy per bit of data transferred. In this paper, the energy efficiency for a wireless transceiver is expressed as a closed form function of transmission time, power consumption for each power state, and frame length.

In Figure 5, there are four power states: TX, RX, Listen, and Doze. The TX, RX, and Listen states are parts of the active mode; the Doze state is the doze mode. Table 1 shows current consumption simulation parameters for different power satates and bandwidth modes [13]. The proposed dynamic energy saving mechanism was evaluated usign a computer simulation with this power model and parameters under the IEEE 802.11 reference channel model [14].

When a wireless device needs to exchange data, the device enters the awake mode. In the awake mode, the device may waste a significant amount of power, even during an idle listening period, if the device does not use power saving techniques. Therefore, wireless consumer electronic devices need efficient power-saving techniques during their awake state. In a wireless receiver, there is a carrier sensing period for idle listening and a non-carrier sensing period for data processing. The energy consumption during the period $\left(t_{0}, t_{i}+1\right)$ can be described as Equation (1), where $E$ is energy in Joules, and $V, I$, and $t$ are the voltage, amperes, and time, respectively.

$$
E_{i+1}=E_{i}+V \times I_{i} \times\left(t_{i}+1-t_{i}\right) .
$$

Hence, the average energy per bit is calculated using Equation (2), where $E_{T}$ is the total energy consumed during time interval $T$, and $B_{T}$ is the total number of successfully transmitted bits during time interval $T$.

$$
E=E_{T} / B_{T}
$$


Thus, the energy consumption for 1 bit during 1 second can be described as the Equaltion (3).

$$
E[J / b / s]=\left(\sum_{i} T_{i} \cdot P_{i}\right) / L_{\text {data }} / T_{\text {total }} .
$$

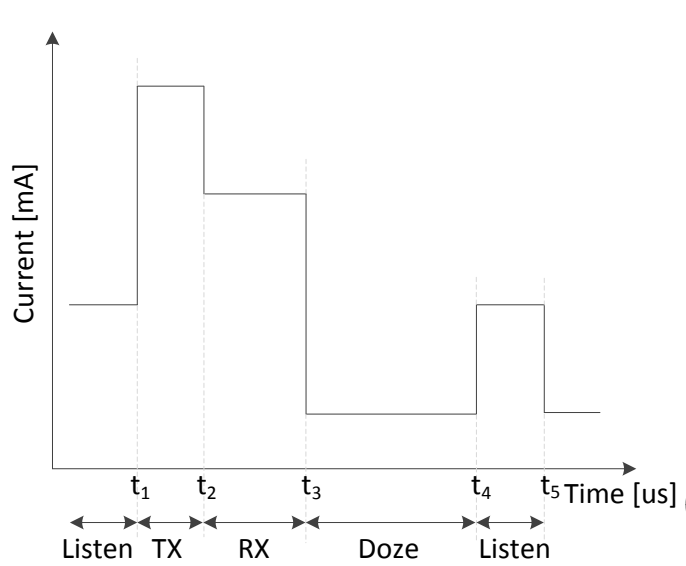

(a)

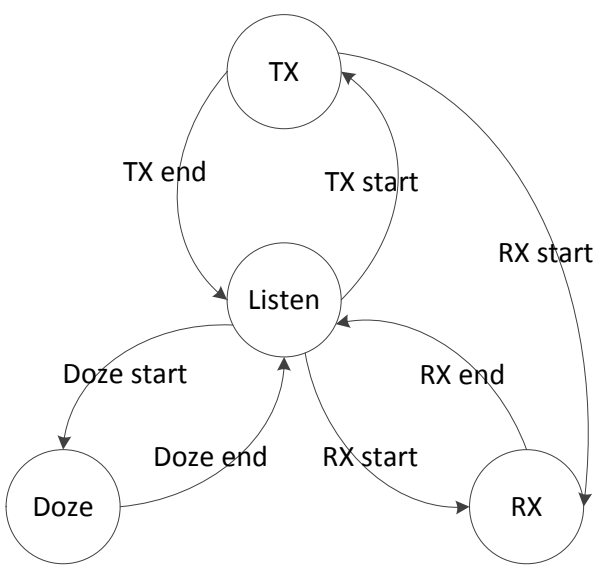

(b)

Figure 5. Energy consumption model: (a) current consumption for power states; and (b) power state flow control; TX: transmission; RX: reception.

Table 1. Current consumption for different power states and bandwidth modes.

\begin{tabular}{cccc}
\hline \multicolumn{4}{c}{ Current Consumption (Voltage = 1.1 V). } \\
\hline Power State & $\mathbf{2 0} \mathbf{~ M H z ~ B W ~}$ & $\mathbf{4 0} \mathbf{~ M H z ~ B W ~}$ & $\mathbf{8 0 ~} \mathbf{~ M H z ~ B W ~}$ \\
\hline PHY TX (mA) & 280 & 285 & 290 \\
PHY RX (mA) & 100 & 110 & 130 \\
Listen (mA) & 50 & 55 & 60 \\
Sleep (mA) & 0.003 & 0.003 & 0.003 \\
MAC TX (mA) & 40 & 60 & 80 \\
MAC RX (mA) & 40 & 60 & 80 \\
\hline
\end{tabular}

Figure 6 shows the simulation results for the power consumption of a WLAN system when the proposed dynamic energy saving mechanism and conventional dynamic bandwidth operation are applied. DES (dynamic energy saving) and DB (dynamic bandwidth) refer to the dynamic energy saving mechanism and the conventional dynamic bandwidth operation, respectively. The proposed DES scheme was compared with the conventional DB scheme for a 20/40/80 MHz signal bandwidth from MCS0 to MCS9 (MCS: modulation and coding scheme). For the settings of this simulation, we assumed that the tested station can adapt the channel bandwidth by utilizing the DB operation for the dynamically changed channel interference conditions. As a result, the DES achieves significantly improved energy efficiency in a narrower channel bandwidth transmission compared with the DB scheme. Figure 7 shows energy consumption over various frame lengths for a $20 \mathrm{MHz}$ channel bandwidth. As a result, a lower frame length consumed more energy due to the increased overhead ratio, such as the inter-frame space, preamble, header, backoff, and control frames. In shorter frame length conditions, a lower rate increased the gap of energy per bit consumption between DES and DB because of the energy saving gain. 


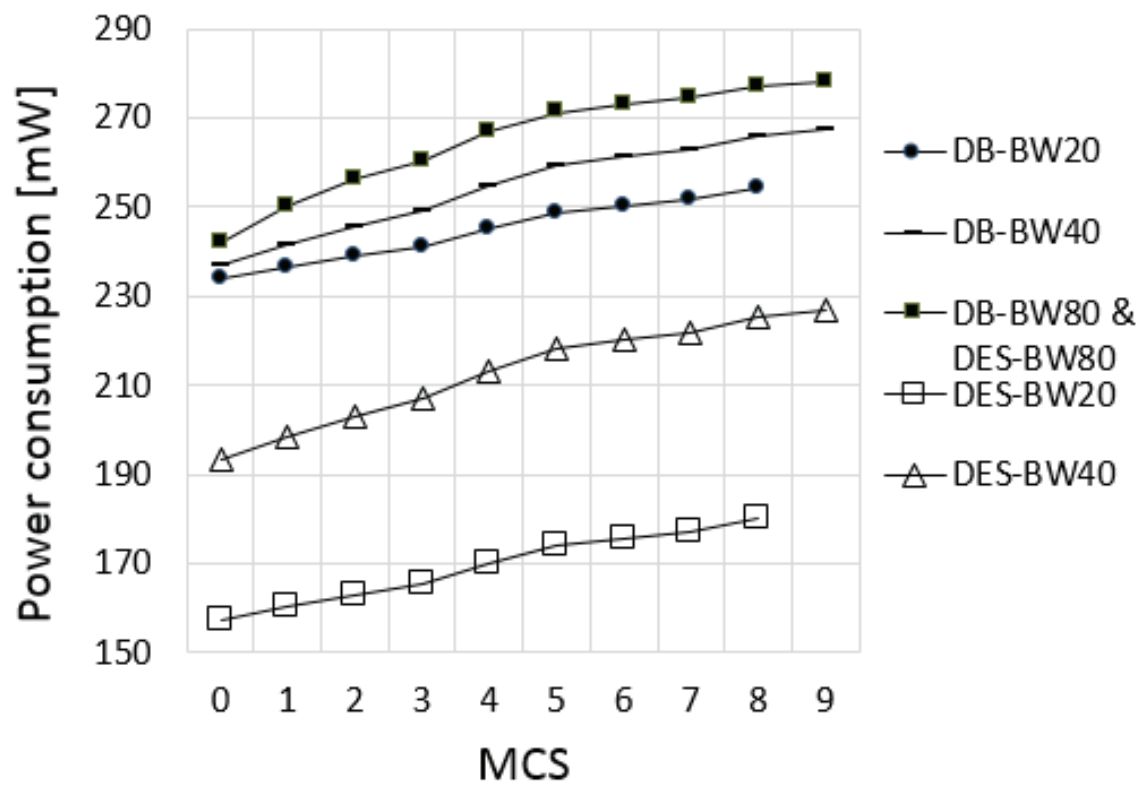

Figure 6. Energy consumption over various parameters: MCS, bandwidth, and operating scheme in the active mode.

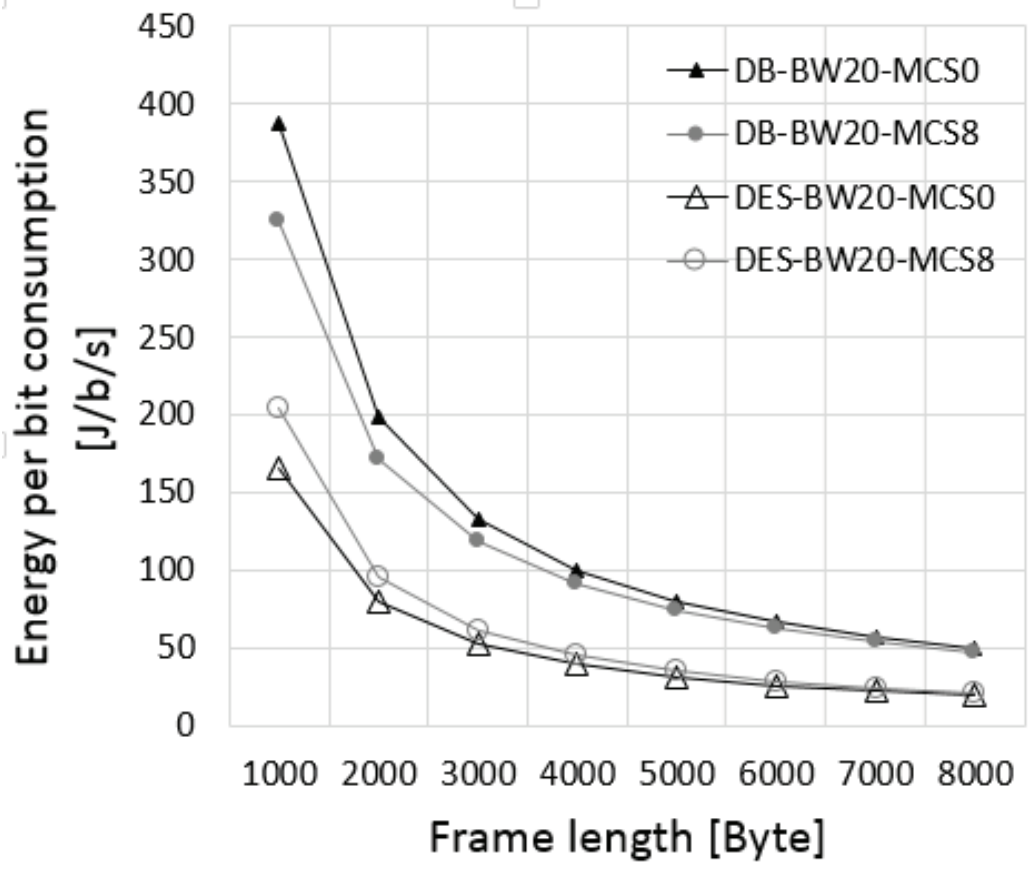

Figure 7. Energy consumption over different frame lengths.

Figure 8 demonstrates energy consumption results for DES and DB when they employ link adaptation. In this simulation, it is assumed that the station adapts the data rates with higher priority for narrower bandwidth when it is necessary to select the channel bandwidth mode. For example, the station selects MCS3 (26 Mbps) in a $20 \mathrm{MHz}$ bandwidth instead of MCS1 (27 Mbps) in a $40 \mathrm{MHz}$ bandwidth if the $40 \mathrm{MHz}$ channel is available. In this simulation, the auto rate fallback (ARF) algorithm is used for the link adaptation. As a result, DES demonstrated significantly improved energy efficiency up to a $234 \mathrm{Mbps}$ data rate. 


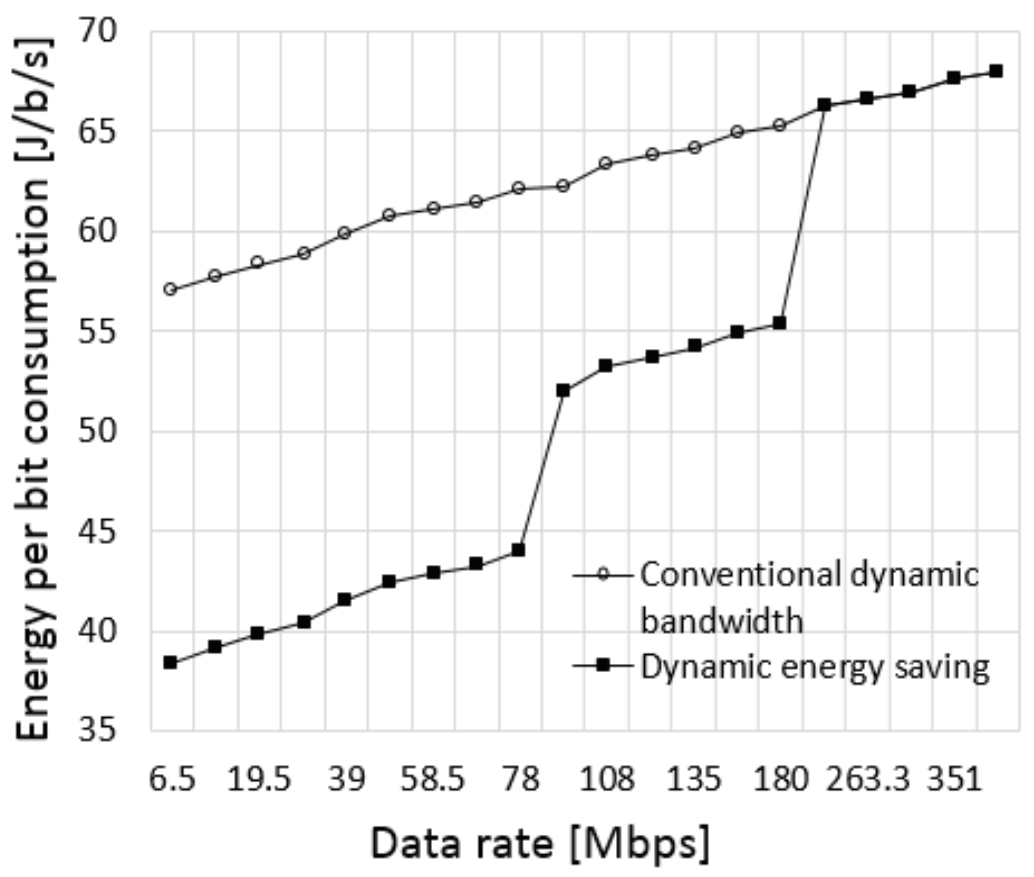

Figure 8. Energy consumption over data rates when using the link adaptation scheme.

\section{Conclusions}

Energy consumption is a major concern of IoTs industries, and energy saving technologies have already been implemented in WLANs. WLAN enabled IoTs require low power operation because the network needs to operate for prolonged periods on restrained power sources. Several IoTs products have the ability to provide Wi-Fi connectivity such as IEEE $802.11 \mathrm{~b} / \mathrm{g} / \mathrm{n}$ [15-18]. Furthermore, IEEE 802.11ah/af supporting multiple sub-channels were designed for low power IoTs applications. This paper proposed an efficient energy saving mechanism for multi-channel IoTs for WLANs. We demonstrated that the multi-channel IoT's energy efficiency is improved by reducing the operating clock frequencies. In this paper, a closed-form analytical model describes the relationship between energy consumption, signal bandwidth, and operating clock frequency. In this paper, it is verified that the energy consumption was reduced by adaptive control of operating clocks even in the active mode. For a further study, this work will be extended to implement and demonstrate the proposed dynamic energy saving mechanism in a real testbed and study transmission strategies for higher energy efficiency.

Conflicts of Interest: The author declares no conflict of interest.

\section{References}

1. Institute of Electrical and Electronics Engineers. IEEE Standard for Information Technology_Telecommunications and Information Exchange between Systems Local and Metropolitan Area Networks-Specific Requirements-Part 11: Wireless LAN Medium Access Control (MAC) and Physical Layer (PHY) Specifications-Amendment 4: Enhancements for Very High Throughput for Operation in Bands below 6 GHz; IEEE Standard 802.11ac; IEEE: Piscataway, NJ, USA, 2013.

2. Institute of Electrical and Electronics Engineers. IEEE Standard for Information Technology_Telecommunications and Information Exchange between Systems_Local and Metropolitan Area Networks_Specific Requirements_Part 11: Wireless LAN Medium Access Control (MAC) and Physical Layer (PHY) Specifications Amendment 5: Television White Spaces (TVWS) Operation; IEEE Standard 802.11ac; IEEE: Piscataway, NJ, USA, 2014. 
3. Institute of Electrical and Electronics Engineers. IEEE Draft Standard for Information Technology-Telecommunications and Information Exchange between Systems_Local and Metropolitan Area Networks_Specific Requirements_Part 11: Wireless LAN Medium Access Control (MAC) and Physical Layer (PHY) Specifications: Amendment—Sub 1 GHz License-Exempt Operation; IEEE P802.11ah; IEEE: Piscataway, NJ, USA, 2014.

4. Goth, G. Next-Generation Wi-Fi: As Fast as We'll Need? IEEE Internet Comput. 2012, 16, 7-9.

5. Sun, W.; Lee, O.; Shin, Y.; Kim, S.; Yang, C.; Kim, H.; Choi, S. Wi-Fi could be much more. IEEE Commun. Mag. 2014, 52, 22-29.

6. Tozlu, S. Feasibility of Wi-Fi Enabled Sensors for Internet of Things. In Proceedings of the 7th International Wireless Communications and Mobile Computing Conference (IWCMC), Istanbul, Turkey, 5-8 July 2011; pp. 291-296.

7. Tozlu, S.; Senel, M.; Mao, W.; Keshavarzian, A. Wi-Fi enabled sensors for internet of things: A practical approach. IEEE Commun. Mag. 2012, 50, 134-143.

8. Perahia, E.; Stacey, R. Next Generation Wireless LANs: $802.11 \mathrm{n}$ and 802.11 ac; Cambridge University Press: Cambridge, UK, 2013.

9. Oppenheim, A.V. Discrete-Time Signal Processing; Pearson Education India: New Delhi, India, 1999; p. 518.

10. Zhang, X.; Shin, K.G. E-mili: energy-minimizing idle listening in wireless networks. IEEE Trans. Mobile Comput. 2012, 11, 1441-1454.

11. Lee, I.G.; Kim, Y.J.; Lee, S.K. Power aware wireless receiver design utilizing carrier sensing based on crosscorrelation. In Proceedings of the 2nd International Conference on Signal Processing and Communication Systems, ICSPCS 2008, Gold Coast, Australia, 15-17 December 2008; pp. 1-2.

12. Zeng, Y.; Pathak, P.H.; Mohapatra, P. A first look at 802.11 ac in action: Energy efficiency and interference characterization. In Proceedings of the 2014 IFIP Networking Conference, Trondheim, Norway, 2-4 June 2014; pp. 1-9.

13. Yu, C.; Yee, J.; Jauh, A.; Hsu, F.; Wang, C.; Huang, R.; Bajko, G.; Wang, J. Energy Efficiency Evaluation and Simulation Model; IEEE 802.11-14/1444r1; IEEE: Piscataway, NJ, USA, 2014.

14. Breit, G.; Sampath, H.; Vermani, S.; Van Nee, R.; Cheong, M.; Kwak, B.; Kim, M.D.; Park, J.J.; Honma, N.; Yasushi, T.; et al. TGac Channel Model Addendum; IEEE 802.11-09/0308r12; IEEE: Piscataway, Nj, USA, 2014.

15. Wi-Fi Alliance. Connect Your Life: Wi-Fi and the Internet of Everything. Available online: http://www.wifi.org/system/files/wp_Wi-Fi_Internet_of_Things_Vision_20140110.pdf (accessed on 28 May 2017).

16. GS 2000. Available online: http:/ /www.gainspan.com/products/gs2000 (accessed on 18 May 2017).

17. TI Simplelink WiFi. Available online: http://www.ti.com/lsds/ti/wireless-connectivity/wi-fi/overview. page (accessed on 20 April 2017).

18. RS9110 Lite-Fi. Available online: http:/ /www.redpinesignals.com/Chipsets/ (accessed on 20 May 2017). 